\title{
ANÁLISIS DE LOS ÍNDICES DE RIESGO DE CALIDAD DE AGUA POTABLE (IRCA) EN BOYACÁ ENTRE 2016-2019
}

\author{
ANALYSIS OF WATER QUALITY RISK INDEX (WQRI) IN BOYACÁ \\ DEPARTAMENT BETWEEN 2016-2019
}

\author{
Diana Lorena Rojas Rodríguez \\ Estudiante Ingeniería Ambiental, 9 Semestre UNAD \\ https://orcid.org/0000-1702-6672 \\ dlrojasro@unadvirtual.edu.co
}

Ramiro Andrés Colmenares Cruz

Ingeniero Ambiental; Esp. Gestión de La Seguridad y Salud en el Trabajo;

M.Sc (c) Gestión y Administración Ambiental (E) Doctorado en Ciencias

Ambientales y Sostenibilidad Docente UNAD.

https://orcid.org/0000-0002-0392-7333

ramiro.colmenares@unad.edu.co

\begin{abstract}
RESUMEN
La presencia de enfermedades gastrointestinales en la población de los 123 municipios del departamento de Boyacá depende de la calidad de agua que suministran las entidades prestadoras del servicio de acueducto. Existen diferentes factores que afectan la prestación de un servicio de calidad, ya sea, por falta de asesoría, acompañamiento o por la inadecuada gestión de la administración. En este sentido el análisis de los acueductos a través de la inspección, vigilancia y control de la calidad de agua da como resultado el índice de riesgo de calidad de agua IRCA, aspecto que favorece su interpretación para buscar estrategias y reducir la probabilidad de que una población presente enfermedades asociadas a su consumo. Así, este trabajo de investigación analizó los reportes del IRCA correspondientes al periodo comprendido entre 2016 y 2019, a través de un comparativo de su variación en el tiempo, además de evaluar su relación con la ocurrencia de enfermedades transmitidas por el agua, lo que logró determinar que gran parte de los municipios de Boyacá, presentan problemas físicos químicos y microbiológicos en el agua potable, principalmente enfocados a la presencia de E. coli, coliformes totales, cloro residual y turbiedad; encontrándose en diferentes rangos
\end{abstract}

DOI: https://doi.org/10.22490/26653176.4315 
de riesgo, lo que empeora el panorama actual para el consumo de agua potable apta para los habitantes de los diferentes municipios.

Palabras clave: Agua potable, Calidad del agua, Enfermedades gastrointestinales, Riesgo

\section{ABSTRACT}

The presence of gastrointestinal diseases in the population of the 123 municipalities of the Boyacá depends on the quality of water supplied by the entities that provide the aqueduct service. There are different factors that affect the provision of a quality service, either due to lack of advice, support or inadequate management by the administration. In this sense, the analysis of the aqueducts through the inspection, surveillance and control of water quality, results in the WQRI water quality risk index, an aspect that favors its interpretation to seek strategies and reduce the probability that a population has diseases associated with its consumption. Thus, this research work analyzed the WQRI time reports corresponding to the period between 2016 and 2019, through a comparison of its variation in it, in addition to evaluating its relationship with the occurrence of diseases transmitted by water, which could determine that a large part of the municipalities of Boyacá have physical, chemical and microbiological problems in drinking water, mainly focused on the presence of E. coli, total coliforms, residual chlorine and turbidity; being in different risk ranges, which worsens the current panorama for the consumption of potable water suitable for the inhabitants of the different municipalities.

Keywords: Drinking water, Water quality, Gastrointestinal diseases, Risk

\section{RESUMEN GRÁFICO}

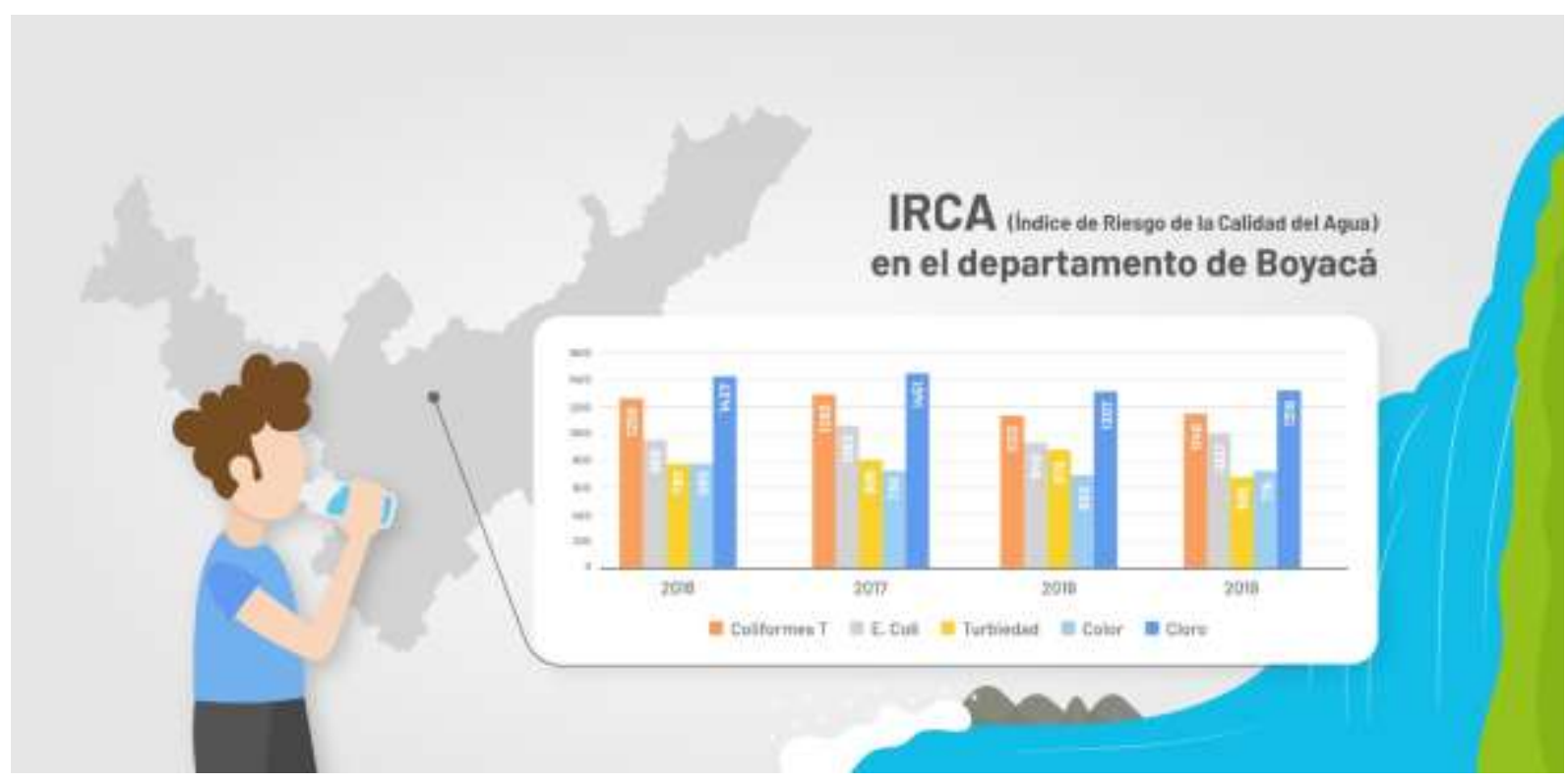

Fuente: Autores 


\section{INTRODUCCIÓN}

Para la Organización Mundial de la Salud OMS (2002), la calidad del agua potable es de interés universal, ya que hace parte esencial para las labores de la cotidianidad de las personas, y en este sentido se debería garantizar un suministro satisfactorio. Así, en julio del 2010 a través de la Resolución 64/292 la ONU (2010), reconoció el derecho humano al agua, y con ello, se consolidaron parámetros de calidad de agua potable, que se caracterizó por ser una estrategia que brinda a los pobladores su apropiada calidad, libre de contaminación, en particular de la materia fecal (OMS, 2012). Así el Departamento Nacional de Planeación DNP (2018) ha evidenciado el aumento de la población colombiana con respecto a acceso de agua potable, lo que ha traído consigo, una mejora en las metas del objetivo de desarrollo sostenible 6 (ODS-6) y por consiguiente una reducción de la pobreza a pesar de que el acceso es significativamente mayor en zonas urbanas que en las zonas rurales (OMS, 2012).

En Colombia, el Índice de Riesgo de Calidad de Agua para consumo humano - IRCA corresponde al grado de ocurrencia de factores relacionadas con el no cumplimiento de las características físicas, químicas, microbiológicas y parasitológicas del agua que conduce a las zonas residenciales. Este indicador es el resultado de asignar el puntaje de riesgo establecido por la Resolución No. 2115 de 2007, cuyo principio se basa en la asignación de valores de riesgo de acuerdo con cada uno de los factores, donde se contempla a E. coli, coliformes totales, turbiedad, y color aparente, como las variables de mayor relevancia en la categorización del nivel bajo, medio y alto de riesgo (García-Ubaque et al., 2018).

Por consiguiente, en el Departamento de Boyacá por medio programas de calidad de agua para el consumo humano, viene desarrollando estrategias enfocadas a mejorar la calidad de vida mediante el suministro de agua potable a los habitantes de los diferentes municipios. Este líquido es captado de diferentes fuentes hídricas tanto superficiales como subterráneas, con el fin de garantizar la distribución del producto, que aseguren que el agua comercializada ofrece óptimas condiciones de consumo (Gobernación de Boyacá, 2018).

El interés de desarrollar la presente investigación surge de la necesidad de evaluar el estado actual de la calidad del agua suministrada, por medio de las empresas prestadoras del servicio en las áreas urbanas y rurales del Departamento de Boyacá, teniendo como visión poder contribuir con el desarrollo regional. Razón por la cual el objetivo de esta investigación fue analizar los índices de calidad de agua potable (IRCA) en el departamento de Boyacá durante el periodo 2016 - 2019.

\section{MATERIALES Y MÉTODOS}

\section{Tipo de estudio}

Esta investigación tuvo un enfoque cualitativo con un diseño descriptivo que de acuerdo con lo propuesto por Barbosa et al. (2013), se caracteriza por tener una particular 
correspondencia en la búsqueda de la problemática y detallar los intereses de la investigación, así, se desarrolla a través de la búsqueda de información secundaria fiable, y genera un panorama holístico desde los autores.

\section{Sitio}

La investigación cubrió los datos de calidad de agua de los acueductos de los 123 municipios de Boyacá, reportados por la Secretaría de Salud, en el periodo comprendido entre 2016 y 2019, en función de los datos del IRCA.

\section{Metodología}

Los datos fueron organizados en una matriz, donde se analizaron parámetros físicos, químicos y microbiológicos del agua para cada municipio, teniendo en cuenta variables como: color aparente, turbiedad, color residual libre, alcalinidad total, calcio, fosfatos, manganeso, molibdeno, magnesio, zinc, dureza total, sulfatos, hierro total, cloruros, nitratos, nitritos, aluminio, fluoruros, COT, coliformes totales y E. coli, siguiendo los puntajes decretos en la tabla 1.

Tabla 1. Puntaje de riesgo de calidad de agua potable asociado a cada característica.

\begin{tabular}{lc}
\hline \multicolumn{1}{c}{ Característica } & $\begin{array}{c}\text { Puntaje } \\
\text { de riesgo }\end{array}$ \\
\hline Color Aparente & 6 \\
Turbiedad & 15 \\
PH & 1.5 \\
Cloro residual libre & 15 \\
Alcalinidad Total & 1 \\
Calcio & 1 \\
Fosfatos & 1 \\
Manganeso & 1 \\
Molibdeno & 1 \\
Magnesio & 1 \\
Zinc & 1 \\
Dureza total & 1 \\
Sulfatos & 1 \\
Hierro total & 1.5 \\
Cloruros & 1 \\
Nitratos & 1 \\
Nitritos & 3 \\
Aluminio (Al ${ }^{3+}$ ) & 3 \\
Fluoruros & 1 \\
COT & 3 \\
Coliformes totales & 15 \\
Escerichia coli & 25 \\
Sumatoria de puntajes & 100 \\
asignados & \\
\hline
\end{tabular}

Fuente: autores 
La anterior tabla logra facilitar determinar los niveles de riesgo (IRCA \%) que se clasifican en inviable sanitariamente $(80.1-100 \%)$, alto (35.1 - 80\%), medio (14.1 - 35), bajo (5.1 - 14) y sin riesgo (menor a 5) que se determinó siguiendo la ecuación 1 :

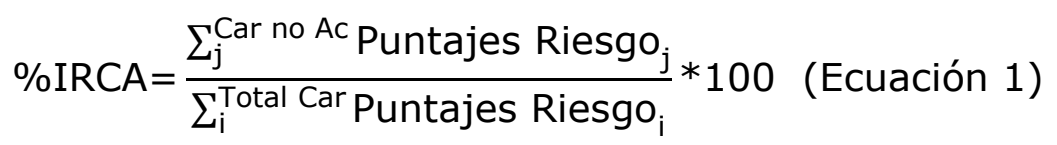

Donde,

Car no ac: Características no aceptables

Total car: Total de Características

\section{Análisis de datos}

La información obtenida durante el periodo 2016 al 2019 se organizó, contrasto y trianguló para observar definir la calidad del agua suministrada en todos los municipios del Departamento de Boyacá durante este lapso. Se elaboró el consolidado para determinar el nivel de riesgo del agua suministrada por las empresas prestadoras del servicio a nivel general tanto en el área rural como urbano. Además, se evaluó si en este periodo se implementaron acciones correctivas y preventivas que han mejorado el servicio prestado por las alcaldías municipales y las juntas de acción comunal. En este sentido, los datos fueron analizados a través de una matriz de doble entrada, con cluster jerárquico (Bootstrap) superior y lateral, agrupando los municipios que mayor similitud tiene con las variables; para esto se utilizaron las librerías gplots y RColorBrewer a través del programa R versión 3.6.3. Los mapas fueron construidos en ArcGIS map.

\section{RESULTADOS Y DISCUSIÓN}

Los resultados obtenidos para el año 2016 se observan en la figura 1 . Se determina que 72 municipios cumplen con lo establecido por la resolución 2115 del 2007, 34 se encuentran en riesgo bajo lo que implica que a pesar de las estrategias desarrolladas por los diferentes acueductos aún no cumplen con los requerimientos necesarios. Por otra parte, 17 municipios se encuentran en riesgo medio y deben mejorar los procesos que se están implementando con el ánimo de ofrecer un servicio que mejore la calidad de vida de los usuarios.

En el consolidado IRCA rural 2016; 11 de los municipios son inviables sanitariamente debido a la falta de gestión realizada por parte de las administraciones municipales y por la falta de capacitación e interés de las administraciones de los acueductos, 69 se encuentran en riesgo alto afectando la salud de los usuarios y desmejorando su calidad de vida, 27 riesgo medio, 11 riesgo bajo; 4 sin riesgo; por otro lado, fue imposible realizar la toma de muestras respectivas en Cubará 


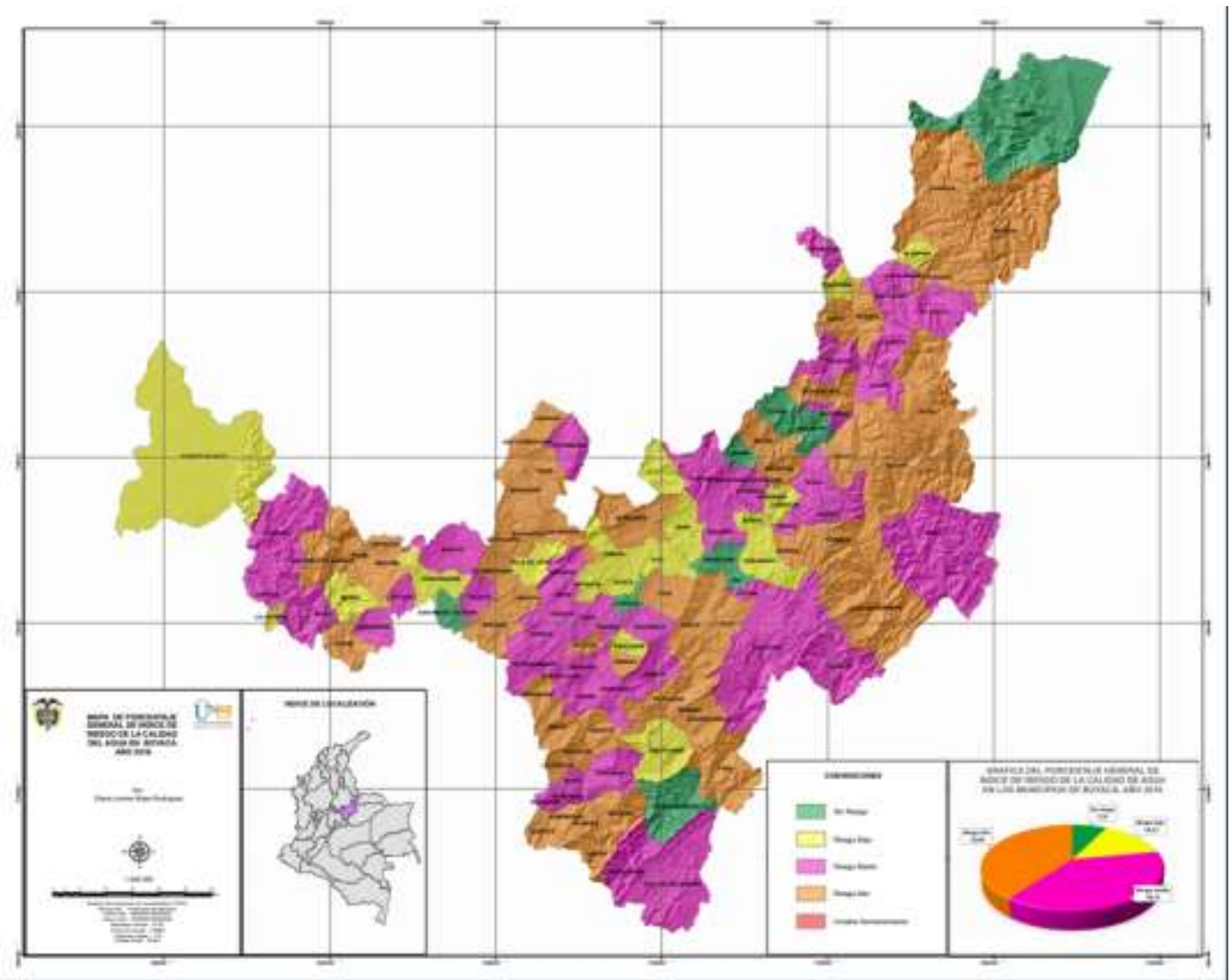

Figura 1. Datos de la información obtenida del Índice de Riesgo de Calidad de Agua IRCA en los municipios del departamento de Boyacá para el año 2016. Fuente: Autores

De acuerdo con el análisis jerárquico del costado izquierdo (Figura 2), se logra identificar que las variables se aglomeran en dos grandes grupos. Uno, y quizás el más importante recoge las variables coliformes totales, cloro residual, E. Coli, turbiedad y color aparente; aspecto que se resalta debido a que es la problemática más recurrente en el análisis fisicoquímico y microbiológico en las muestras de agua. Así de acuerdo con el análisis jerárquico superior se identificaron cinco grupos en los que se conjugan todos los municipios del departamento de Boyacá. Para el primer grupo (1) se resalta a Tunja, Duitama, Sotaquirá y Turmequé como los de mayor relación positiva con el primer aglomeramiento del análisis jerárquico izquierdo; aspecto que puede atribuirse a que el número de muestreos durante el año 2016 fue mayor en estas dos grandes ciudades (Tunja y Duitama) en comparación a los demás Municipios, por lo que la probabilidad de que las muestras salieran con anormalidades fisicoquímicas y microbiológicas era mayor. Por lo contrario, el conglomerado 2, muestra una gran homogeneidad de cumplimiento en los parámetros de calidad de agua. Mientras que en el aglomerado número 3 , se agrupan municipios que en su mayoría presentan problemas con cloro residual, turbiedad y en menor proporción con E. Coli y coliformes totales. A su vez, el grupo 4 quien reúne los municipios con una mayor recurrencia en los problemas del grupo 3, además de color aparente y turbiedad. Finalmente, el último grupo (5) en el que predominan problemáticas de coliformes totales, cloro residual y E. coli. 


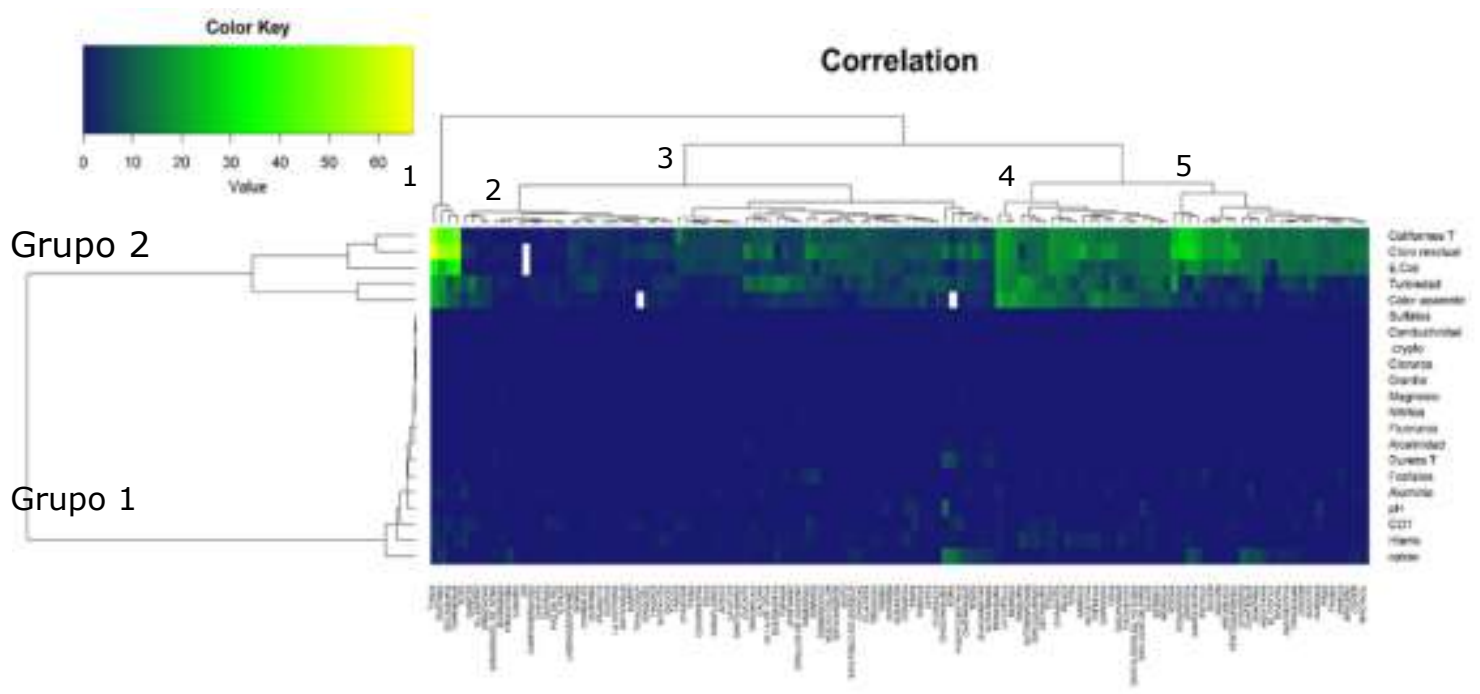

Figura 2. Matriz de doble entrada para el análisis de parámetros fisicoquímicos y microbiológicos del agua potable en el periodo 2016 en los municipios del Departamento de Boyacá. El análisis jerárquico externo indica el agrupamiento de variables y municipios con el método de Ward. Fuente: Autores

En este sentido, los diferentes departamentos de Colombia presentan un desempeño de calidad de agua dependientes de factores como la infraestructura, la fuente de abastecimiento, el manejo en las plantas, y su curso hasta la fuente final, lo que pone a Boyacá para el año 2017 como un departamento con riesgo medio, que junto con otros departamentos suman el 42,2\% (Minsalud, 2019). De acuerdo con lo anterior, fue el mejor resultado después de lo obtenido en años anteriores, donde cerca del $77 \%$ de los municipios de Boyacá, se encontraban con IRCA en riesgo alto (Dueñas-Celis et al., 2018), aspecto que para Bai et al. (2012), favorece el padecimiento de enfermedades agudas y crónicas como patologías parasitológicas, intoxicaciones, alteraciones en el transito digestivo entre otras. En el mapa generado con los datos del IRCA municipal 2017 se puede observar que son muy pocos los municipios que cumplen los requerimientos planteados de acuerdo con la resolución 2115 del 2007 y el mayor porcentaje de municipio tienen un riesgo alto y riesgo medio, por esta razón se requiere la implementación de estrategias que mejoren el suministro de la calidad del agua en el departamento de Boyacá. 


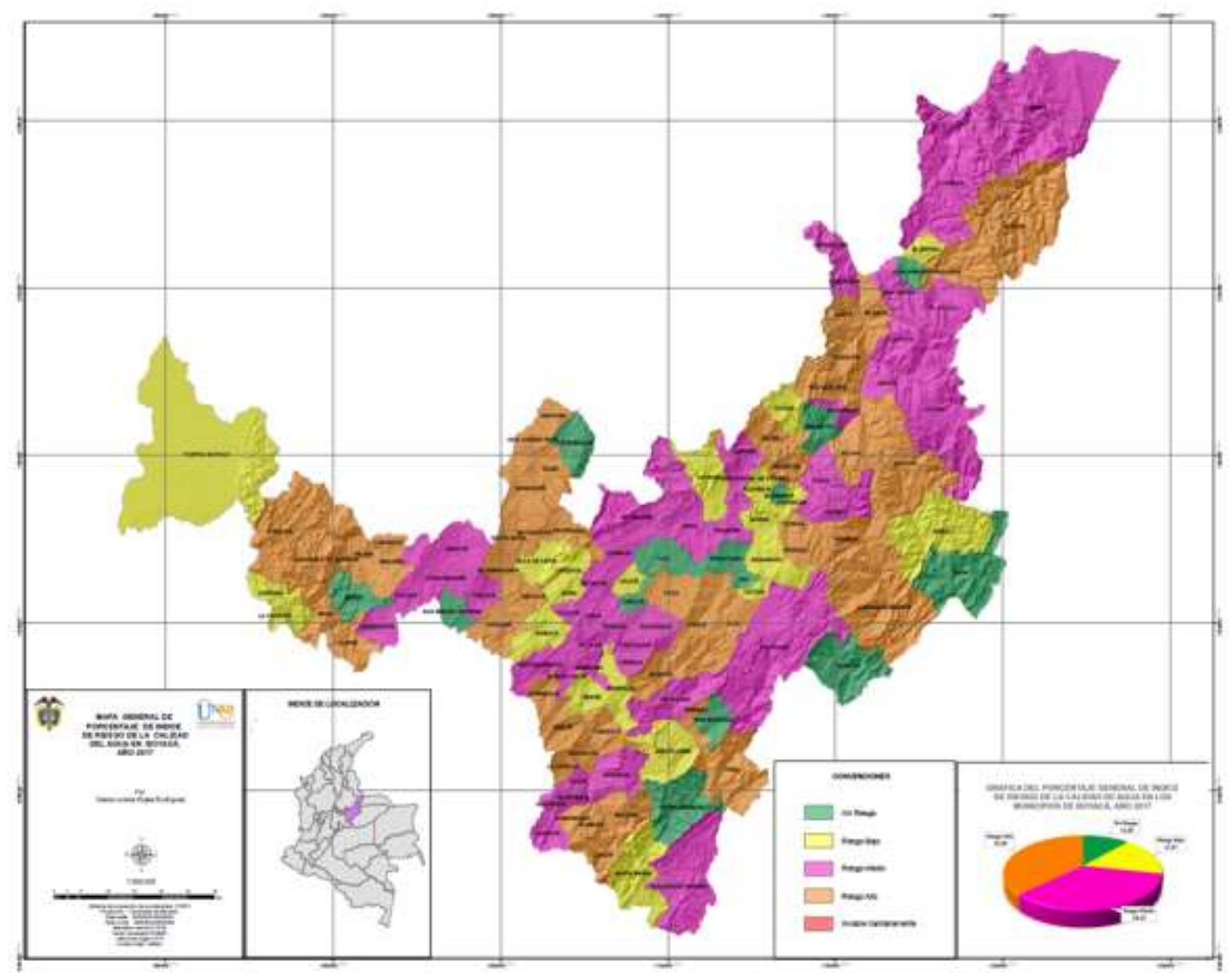

Figura 3. Datos de la información obtenida del Índice de Riesgo de Calidad de Agua IRCA en los municipios del departamento de Boyacá para el año 2017. Fuente: Autores

Para el caso del periodo 2017, Tunja, Turmequé y Duitama, presentan los más altos índices de irregularidad en coliformes totales, mientras que los dos primeros más el municipio de Moniquirá, manifiestan problemas en cloro residual. Es así como en el análisis jerárquico muestra un aumento significativo en la intensidad de la clave colorimétrica en las variables del primer grupo del análisis jerárquico del costado izquierdo en comparación con el año 2016, lo que evidencia una mayor problemática en la calidad físicoquímica y microbiológica del agua potable, principalmente en los municipios que se encuentran en los grupos 1 y 2 , mientras que una fuerte amenaza en los municipios del grupo 4. Conjuntamente, se identificaron leves irregularidades con fosfatos, calcio y aluminio en todos los grupos, pero principal, ente los grupos 3 y 4 (Figura 4). 


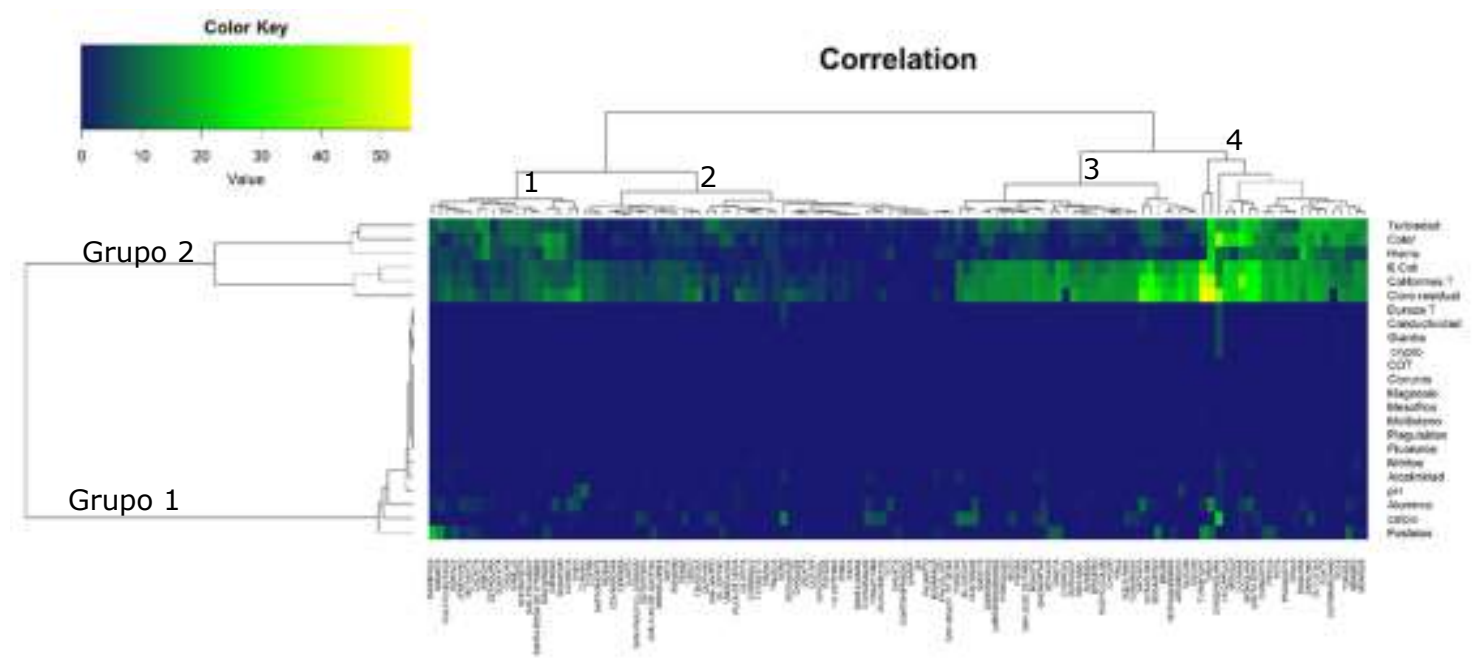

Figura 4. Matriz de doble entrada para el análisis de parámetros fisicoquímicos y microbiológicos del agua potable en el periodo 2017 en los municipios del Departamento de Boyacá. El análisis jerárquico externo indica el agrupamiento de variables y municipios con el método de Ward. Fuente: Autores

De acuerdo con los datos obtenidos, se logra evidenciar un leve aumento en algunos de los índices de riesgo de la calidad de agua en comparación con el año 2016, generando una mayor preocupación para la salud pública de los usuarios de los distintos acueductos municipales. En este sentido, el Instituto Nacional de Salud (INS, 2018), resaltó que el departamento de Boyacá no reportó datos para este año a nivel de las entidades nacionales, lo que se muestra como una gran desventaja ante los panoramas comparativos con los demás departamentos. Sin embargo, la Secretaría de Salud de Boyacá consolida todos los datos de calidad de agua, permitiendo estar disponibles para las entidades que lo requieran.

A nivel general se puede observar que frente al año inmediatamente anterior, el IRCA municipal empeoró en sus condiciones sanitarias porque únicamente seis municipios se encuentran sin riesgo por lo tanto se observa la falta de compromiso por parte de las administraciones municipales en ofrecer un servicio de buena calidad. 


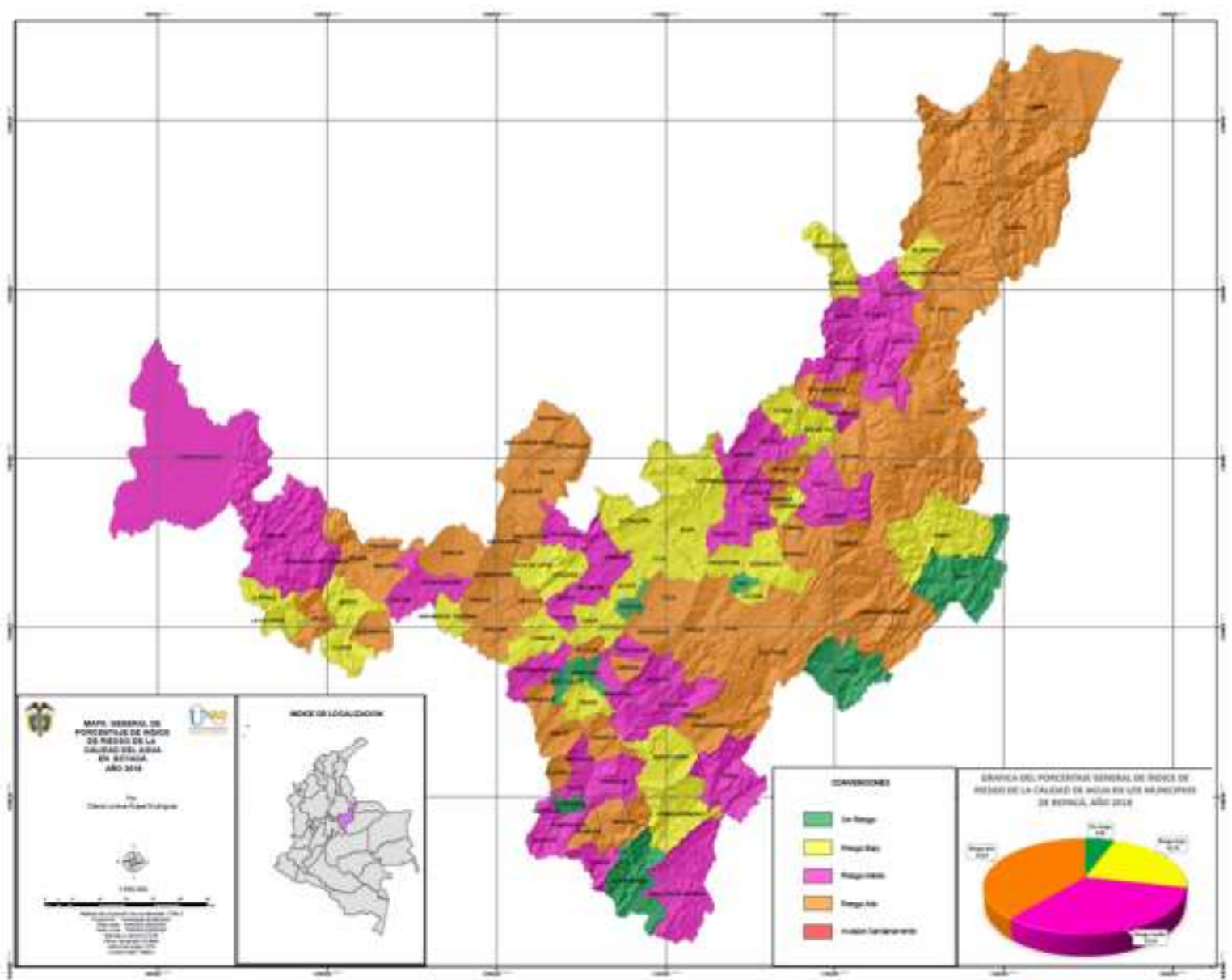

Figura 5. Datos de la información obtenida del Índice de Riesgo de Calidad de Agua IRCA en los municipios del departamento de Boyacá para el año 2018. Fuente: Autores

Para el caso de 2018 (Figura 5), se presenta un comportamiento muy similar al obtenido en el periodo 2017, aspecto que se puede evidenciar en la recurrencia de irregularidad por parte de las muestras de agua potable en los municipios de Turmequé y Tunja, pero, además, para todos los municipios del grupo 1, de acuerdo con el análisis jerárquico del costado superior, en relación con las variables del segundo conglomerado del análisis jerárquico izquierdo. En este mismo sentido, se presenta una problemática marcada en los municipios agrupados en el conglomerado 3, principalmente para E. coli, Coliformes T. y Cloro residual, mientras que una leve incidencia en los municipios de los conglomerados 4 y 5 . Conjuntamente, se evidencia anomalías en la presencia de Hierro, Aluminio, Fosfatos y Calcio en los grupos 1, 3 y 4 (Figura 6). 


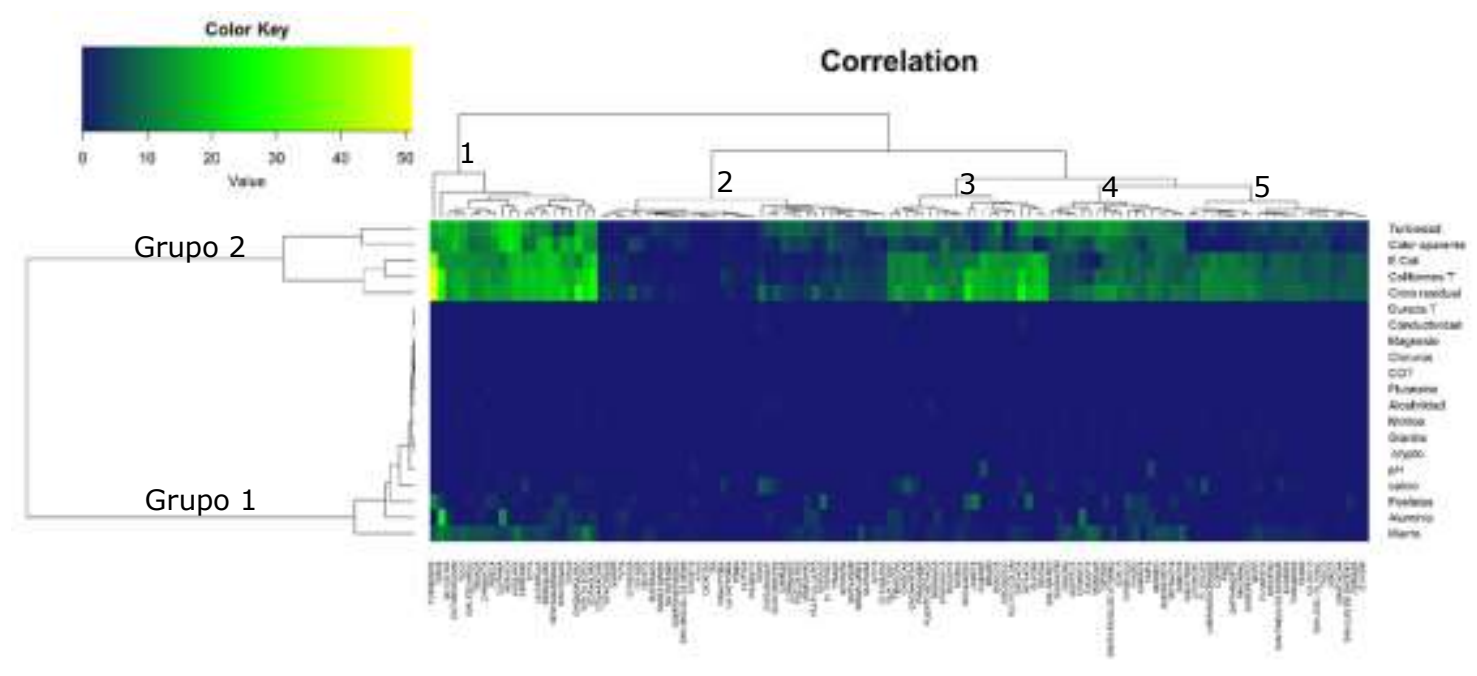

Figura 6. Matriz de doble entrada para el análisis de parámetros fisicoquímicos y microbiológicos del agua potable en el periodo 2018 en los municipios del Departamento de Boyacá. El análisis jerárquico externo indica el agrupamiento de variables y municipios con el método de Ward. Fuente: Autores

De acuerdo con la FAO (2013), la calidad de agua para el consumo humano ha venido perdiendo calidad en términos de sus características físicas, químicas y microbiológicas; parámetros que se agravan en la medida en que pasan los años, dato que es correlacional con los datos analizados en esta investigación. En este sentido Martínez-Austria y PatiñoGómez (2012), resalta que tanto la disponibilidad como las características del agua, se relacionan directamente con las condiciones climáticas de cada una de las regiones, por lo que como consecuencia del cambio climático se agrava calidad y cantidad hídrica en las zonas de captación.

En el desarrollo del mapa IRCA municipal se observa que aumentaron la cantidad de municipios que se encuentran sin riesgo, esto se debe a la intervención realizada por parte de las diferentes entidades gubernamentales, sin embargo, aún se tienen muchos municipios en riesgo alto, razón por la cual no se puede bajar la guardia y seguir gestionando las medidas preventivas necesarias con el ánimo de mejorar la calidad del servicio (Figura 7). 


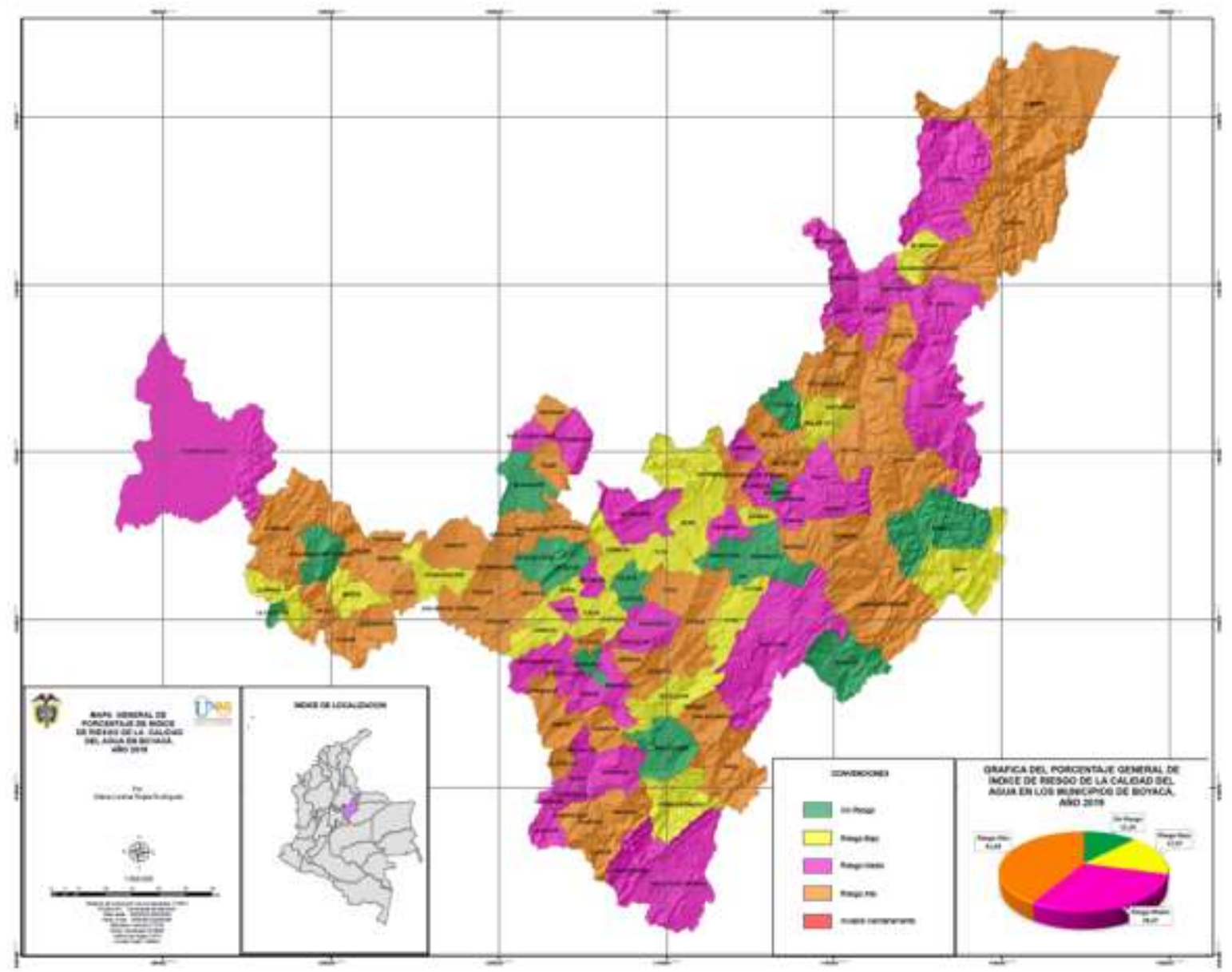

Figura 7. Datos de la información obtenida del Índice de Riesgo de Calidad de Agua IRCA en los municipios del departamento de Boyacá para el año 2019. Fuente: Autores

A diferencia de los años anteriores, los resultados del 2019 mostraron una modificación en el agrupamiento de las variables de acuerdo con el análisis jerárquico del costado izquierdo, lo que se puede atribuir a la significativa irregularidad en la calidad del agua potable en relación con las variables del grupo 1 , correspondientes a $E$. coli, coliformes $\mathrm{T}$. y Cloro residual, principalmente para los municipios de los conglomerados 1 y 2 del análisis jerárquico superior. Sin embargo, el grupo de municipios 1, muestra una marcada irregularidad en variables del conglomerado izquierdo 1 y 3, correspondientes a Turbiedad, Color aparente, Hierro, Cloro residual, Coliformes totales y $E$. coli., lo que lo hace completamente diferentes de los demás grupos. En este sentido, los municipios del grupo 3, manifiestan las mejores características de calidad de agua del departamento, mientras que el grupo 4, una irregularidad media (Figura 8). 


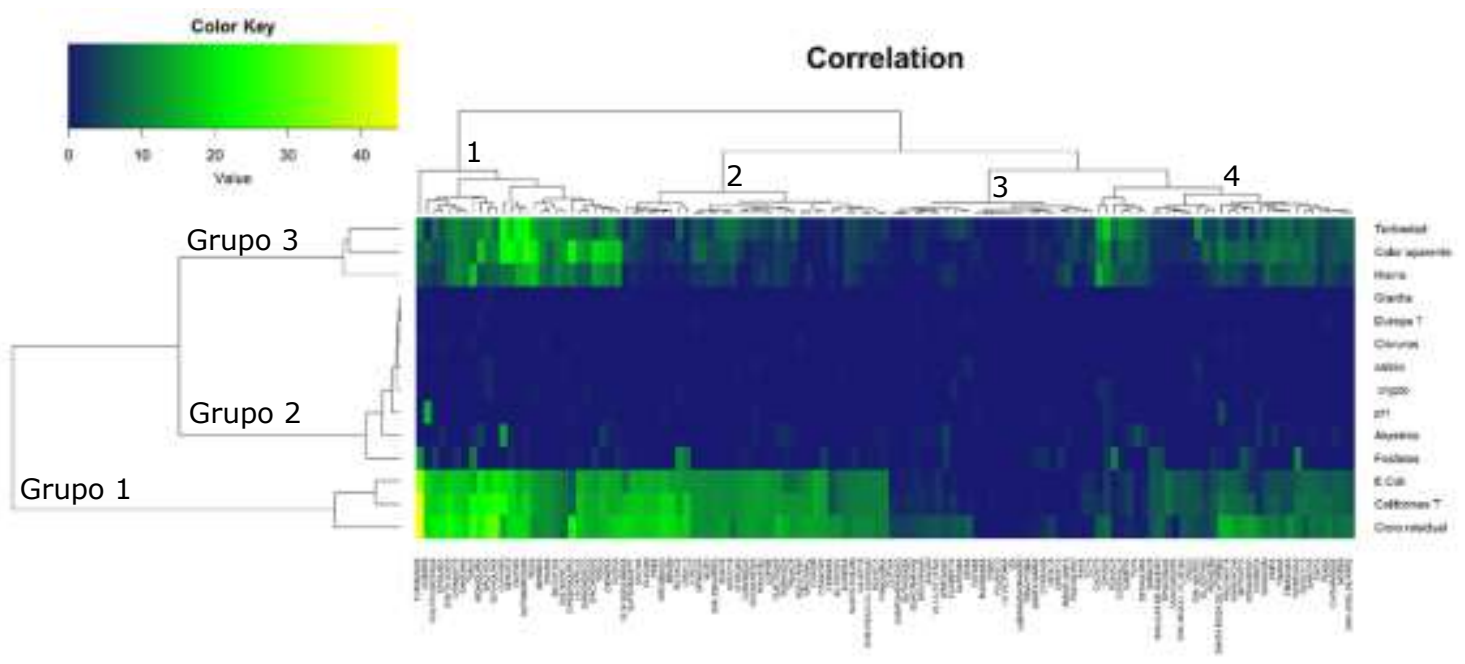

Figura 8. Matriz de doble entrada para el análisis de parámetros fisicoquímicos y microbiológicos del agua potable en el periodo 2019 en los municipios del Departamento de Boyacá. El análisis jerárquico externo indica el agrupamiento de variables y municipios con el método de Ward. Fuente: Autores

Los datos analizados en esta investigación resaltan la preocupante modificación constante de las características físicas, químicas y microbiológicas del agua, lo que trae consigo problemáticas de salud pública como se mencionó anteriormente. De acuerdo con Minsalud (2019), la calidad de agua en el departamento de Boyacá empeora a través de los años, lo que puede ser el resultado de las instalaciones del tratamiento de agua, su estructura de conducción e incluso, los centros de disposición final.

\section{CONCLUSIONES}

Gran parte del estado, calidad, cantidad y continuidad del recurso hídrico en el departamento de Boyacá depende de los entes gubernamentales, por lo tanto, para mejorar la calidad de agua que se suministra es necesario articular todos los entes para que diseñen mejores propuestas de optimización de sistemas de tratamiento que garanticen su captación, tratamiento, distribución y almacenamiento.

La mayoría de los sistemas de tratamiento que se encuentran obsoletos tanto en el área urbano como rural, son por que se han diseñado sin tener en cuenta la calidad del agua de las fuentes abastecedoras en tiempo seco y de lluvia; por lo cual se deberán verificar los proyectos, para que sean planeados de tal forma que suplan y garanticen la calidad de agua para consumo humano y por ende la salud de las poblaciones. 


\section{CONTRIBUCIÓN DE LA AUTORÍA}

Primer autor: metodología, investigación, análisis de datos, conceptualización, escritura, borrador original. Segundo autor: investigación, conceptualización, análisis de datos, escritura, revisión y edición.

\section{AGRADECIMIENTOS}

Los Autores expresan un reconocimiento de agradecimiento a la Universidad Nacional Abierta y a Distancia UNAD, a la Escuela de Ciencias Agrícolas Pecuarias y del Medio Ambiente ECAPMA ZCBOY y a la Secretaria de Salud Departamental de Boyacá que permitió acceder a la información correspondiente al IRCA, con la cual fue posible la realización de esta investigación.

\section{REFERENCIAS}

Bai, X., Nath, I., Capon, A., Hasan, N. y Jaron, D. (2012). Health and wellbeing in the changing urban environment: complex challenges, scientific responses, and the way forward. Current Opinion in Environmental Sustainable. 4(4): 465-472. http//doi.org/10.1016/j.cosust.2012.09.009

Barbosa, J., Barbosa, J., y Villabona, M. (2013). Revisión y análisis documental para estado del arte: una propuesta metodológica desde el contexto de la sistematización de experiencias educativas. Investigación Bibliotecológica: Archivonomía Bibliotecología e Información. 27(61): 83-105.

Dueñas-Celis, M., Dorado-González, L., Espinosa-Macana, P. y Suescún-Carrero, S. (2018). Índice de riesgo de calidad de agua para consumo humano en zonas urbanas del departamento de Boyacá - Colombia, 2004-2013. Revista Facultad Nacional de Salud Pública. 36(3): 101-109. http//doi.org/10.17533/udea.rfnsp.v36n3a10

García-Ubaque, C., García-Ubaque, J., Rodríguez-Miranda, J., Pacheco-García, Robinson. y García-Vaca, M. (2018). Limitaciones del IRCA como estimador de calidad del agua para consumo humano. Revista de Salud Pública. 20(2): 204-207. https://doi.org/10.15446/rsap.v20n2.65952

Gobernación de Boyacá. (2018). Ordenamiento Territorial Departamental de Boyacá OTDB. Recuperado de https://www.dapboyaca.gov.co/wpcontent/uploads/2018/09/DIMENSIONFUNCIONAL SERVICIOS INFRAESTRUCTURA.pdf

Instituto Nacional de Salud INS. (2018). Boletín de vigilancia de calidad de agua para consumo humano 2018. Recuperado de https://www.ins.gov.co/sivicap/Documentacin\%20SIVICAP/1.\%20Boletin\%20Enero \%202018.pdf 
Martínez-Austria, P. \& Patiño-Gómez. (2012). Efectos del Cambio Climático en la disponibilidad de Agua en México. Tecnologías y Ciencias del Agua. 3(1): 5-20.

Ministerio de Salud y Protección Social - Minsalud. (2019). Informe nacional de la calidad de agua para consumo humano INCA 2017. Recuperado de https://www.minsalud.gov.co/sites/rid/Lists/BibliotecaDigital/RIDE/VS/PP/SA/calida d-del-agua-inca-2017.pdf

Ministerio de Salud y Protección Social - Minsalud. (2019). Boletín de vigilancia de la calidad de agua para consumo humano. Recuperado de: https://www.ins.gov.co/sivicap/Documentacin\%20SIVICAP/boletin-vigilanciacalidad-agua-mayo-2019.pdf

Organización Munidial de la Salud OMS (2002). Agua para la salud: un derecho humano. Recuperado de https://www.who.int/mediacentre/news/releases/pr91/es/

Organización Mundial de la Salud OMS. (2017). 2100 millones de personas carecen de agua potable en su hogar y más del doble no disponen de saneamiento básico. Recuperado de https://www.who.int/es/news-room/detail/12-07-2017-2-1-billionpeople-lack-safe-drinking-water-at-home-more-than-twice-as-many-lack-safesanitation

Organización Mundial de la Salud OMS. (2012). Informe del GLAAS de 2012. Análisis y evaluación mundial del saneamiento y el agua potable de ONU-Agua. El reto de ampliar y mantener los servicios. 112ppp. Recuperado de https://apps.who.int/iris/bitstream/handle/10665/91344/9789243503363 spa.pdf?s equence $=1$

Organización de las Naciones Unidas para la Agricultura y la Alimentación FAO. (2013). Afrontar la escasez del agua. Un marco de acción para la agricultura y la seguridad alimentaria. Recuperado de http://www.fao.org/3/a-i3015s.pdf

Organización de las Naciones Unidas ONU. (2010). Decenario Internacional para la acción "El agua fuente de vida". Recuperado de https://www.un.org/spanish/waterforlifedecade/human right to water.shtml 